\title{
Artificial molecular quantum rings: Spin density functional theory calculations
}

\author{
L. K. Castelano, ${ }^{1}$ G.-Q. Hai, ${ }^{1}{ }^{*}$ B. Partoens, ${ }^{2}$ and F. M. Peeters ${ }^{2, \dagger}$ \\ ${ }_{1}^{1}$ Instituto de Física de São Carlos, Universidade de São Paulo, 13560-970, São Carlos, SP, Brazil \\ ${ }^{2}$ Department of Physics, University of Antwerp, Groenenborgerlaan 171, B-2020 Antwerp, Belgium
}

(Received 15 June 2006; published 17 July 2006)

\begin{abstract}
The ground states of artificial molecules made of two vertically coupled quantum rings are studied within the spin density functional theory for systems containing up to 13 electrons. Quantum tunneling effects on the electronic structure of the coupled rings are analyzed. For small ring radius, our results recover those of coupled quantum dots. For intermediate and large ring radii, new phases are found showing the formation of new diatomic artificial ring molecules. Our results also show that the tunneling-induced phase transitions in the coupled rings occur at much smaller tunneling energy as compared to those for coupled quantum dot systems.

DOI: 10.1103/PhysRevB.74.045313

PACS number(s): 73.21.La, 05.30.Fk, 73.23.Hk, 85.35.Be
\end{abstract}

\section{INTRODUCTION}

The realization of electronic structures by design is one of the ultimate goals of nanoscience. By varying the size and geometry of nanocrystallites, it is possible to tune and control the confined quantum states and thus the electronic, magnetic, and optical properties. A very successful example of such systems are semiconductor quantum dots and molecules. These artificial atoms and molecules ${ }^{1}$ exhibit shellfilling and new molecular many-body states which have been confirmed experimentally. These structures can be used as more efficient lasers and as hosts for storing quantum information, i.e., qubit, because of their atomiclike properties.

Quantum rings (QRs) are known for the Aharonov-Bohm effect and its persistent current ${ }^{2}$ where the phase rigidity of the electron wave leads to quantum oscillations in the current. Such nanometer-sized rings are artificial benzene analogues. Experimentally there have been several approaches to fabricate ring structures ranging from top-down approaches, e.g., nanolithography (e.g., atomic force microscope patterning), ${ }^{3}$ to bottom-up approaches as straininduced self-organization ${ }^{4}$ and droplet molecular beam epitaxy (MBE). ${ }^{5}$ The bottom-up approach has been used recently for nanostructured assembly of quantum ring complexes as double rings ${ }^{6}$ and vertically coupled rings. ${ }^{7}$ Due to the ring geometry, these ring complexes open a new route for measurement of quantum interference effects and for novel many-body states.

In the present work we study two vertically coupled rings which exhibit new molecular many-body states, hitherto not found in other systems. The tunability of the ring radius and the inter-ring distance leads to a very rich variety of manyelectron ground states. In previous theoretical studies for single rings, Simonin et al. ${ }^{8}$ showed that a displaced parabolic model is suitable for describing the confinement potential for a self-assembled semiconductor QR. Many-body spectra of single QRs have been obtained using exact diagonalization techniques ${ }^{9}$ and spin-density-functional theory. ${ }^{10}$ A study of the persistent current in QRs for up to six electrons showed that the current-spin-density-functional theory provides a suitable tool for describing the physics related to the Aharonov-Bohm effect. ${ }^{11}$ Very recently, theoretical studies were reported on the single-electron spectrum in two ver- tically coupled QRs (Refs. 12 and 13) and of few electron eigenstates of concentric double QRs. ${ }^{14}$

Two vertically coupled quantum rings (CQRs) form a new type of artificial molecule (AM) where the ring radius together with the inter-ring distance are new tunable parameters providing new degrees of freedom to modulate and control the electronic structure of the artificial ring-shaped "molecules." In the present work, we apply the wellestablished spin-density functional theory to study the ground state configurations or phases of few-electron CQRs. Full phase diagrams of these new quantum ring AMs are obtained up to 13 electrons.

\section{THEORETICAL MODEL}

The electrons are confined in a plane where the confinement potential is modeled by a displaced parabolic function $V(r)=\frac{1}{2} m^{*} \omega_{0}^{2}\left(r-r_{0}\right)^{2}$, where $\mathbf{r}=(x, y)=(r, \theta), \omega_{0}$ is the confinement frequency and $r_{0}$ is the radius of the ring. The tunneling between the two stacked identical rings in the $z$ direction occurs through a potential $V(z)$ modeled by two coupled symmetric GaAs quantum wells with a finite barrier height $V_{0}=250 \mathrm{meV}$ and a well width of $W=120 \AA$. For these parameters we obtain for the energy splitting $\Delta=22.86$ $\times \exp [-d(\AA) / 13.455] \mathrm{meV}$ between the bonding and antibonding states when the two wells are separated by a distance $d$.

We use the Kohn-Sham orbitals $\psi_{n m \sigma}(\mathbf{r})$ $=\exp (-i m \theta) \phi_{n m \sigma}(r) Z(z)$ to express the density and groundstate energy. The eigenfunctions $\phi_{n m \sigma}(r)$ are expanded in the Fock-Darwin basis to solve the Kohn-Sham equation. The contribution from higher excited states is neglected because the confinement in the $z$ direction is much stronger than that in the plane. Therefore the motion in the $z$ direction may be assumed to be decoupled from the in-plane motion. The total density in the rings is $\rho(r)=\sum_{\sigma} \sum_{n, m}\left|\phi_{n m \sigma}(r)\right|^{2}$ where $\sigma$ $=(\uparrow, \downarrow)$ being the $z$ component of the electron spin. Following Ref. 15, we approximate the density in the $z$ direction by $\delta$ functions at the center of the quantum wells. The exchange-correlation energy is treated within the local density approximation using the Tanatar-Ceperley ${ }^{16}$ functional. The ground-state energy and the corresponding electronic 


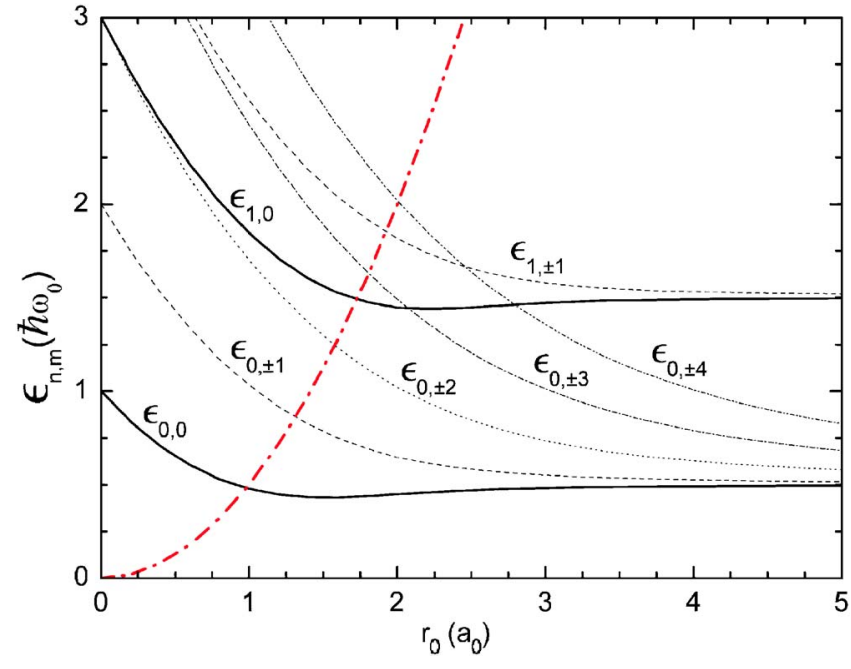

FIG. 1. (Color online) Electron energy levels $\epsilon_{n, m}$ in a single quantum ring as a function of the ring radius. The potential height in the center of the ring is indicated by the thick dotted-dashed line.

configuration of the system are determined by comparing the total energies of all possible configurations. The ground-state phases are labeled by three quantum numbers $\left(S_{z}, M_{z}, I_{z}\right)$ : total spin $S_{z}$, total angular momentum $M_{z}$, and the isospin quantum number $I_{z}$, which is the difference between the number of electrons in the bonding state and in the antibonding state divided by 2 . For the CQRs, the ground-state phases are found for different inter-ring distances (tunneling energy $\Delta)$ and ring radii. In the limits of small and large inter-ring distance $d$, the results for single $\mathrm{QRs}^{10}$ are recovered and in the limit of small ring radius $\left(r_{0} \rightarrow 0\right)$, the previous results for two coupled quantum dots ${ }^{15,17}$ (CQDs) are obtained. The energy levels $\epsilon_{n, m}$ of a single quantum ring are shown in Fig. 1 as a function of the ring radius (in unit $\mathrm{a}_{0}=\sqrt{\hbar / m^{*}} \omega_{0}$ ). The energy levels are labeled by the radial quantum number $n$ $=0,1,2, \ldots$, and the angular quantum number $m$ $=0, \pm 1, \pm 2, \ldots$. As compared to the quantum dot case, in a ring the degeneracy of, e.g., the states $\epsilon_{0, \pm 2}$ and $\epsilon_{1,0}\left(\epsilon_{0, \pm 3}\right.$ and $\epsilon_{1,1}$, etc.) is lifted. With increasing ring radius, the energy difference $\epsilon_{1,0}-\epsilon_{0, \pm 2}$ increases and tends to a constant $\hbar \omega_{0}$ at large $r_{0}$. The effect of lifting the degeneracy becomes significant for systems with nine electrons or more when the energy levels $\epsilon_{1,0}$ and $\epsilon_{0, \pm 2}$ become occupied. Notice that the lowlying energy levels of the $m=0$ orbitals exhibit a minimum as a function of the ring radius. This effect can be understood qualitatively by looking at the potential height $m^{*} \omega_{0}^{2} r_{0}^{2} / 2$ in the center of the ring that is indicated by the thick dot-dashed curve in Fig. 1. On the left-hand side of this curve, the system behaves similar to a quantum dot and to the right of it, a quantum ring starts to form. The local minimum in the $m$ $=0$ curves occurs at this crossover regime and will affect the ground-state phases for CQRs with 12 and 13 electrons with intermediate ring radius.

We want to stress that the above theory is based on the envelope function approach and the local-spin-density approximation (LSDA). These approximations are valid in the present study for the coupled quantum rings. For a confinement strength of $\hbar \omega_{0}=5 \mathrm{meV}$, the corresponding typical length scale is $\mathrm{a}_{0} \simeq 15 \mathrm{~nm}$ in the case of GaAs. ${ }^{3}$ We show results for a diameter up to $2 \times 5 \times \mathrm{a}_{0}=150 \mathrm{~nm}$. These scales are still much larger than the interatomic distances, therefore the effective mass approximation is valid. Moreover, we have chosen for a LSDA-density functional theory (DFT) approach to study the many-electron states of the CQRs. This approach was used before successfully to describe the energy levels in a single quantum dot of comparable size ${ }^{18}$ and worked surprisingly well even for systems of two and three electrons. Ferconi and Vignale ${ }^{18}$ compared the exact result for the ground-state energy of a two-electron quantum dot with a DFT calculation. The accuracy is found to be better than $3 \%$. Therefore we believe that claims we make that are based on the LSDA-DFT approach are justified. We did not implement a higher functional, because it would not reveal new physics. Our confinement potential is anyhow a model system for two coupled rings.

\section{NUMERICAL RESULTS AND DISCUSSIONS}

The stability region of the different ground states are summarized in phase diagrams in the $\Delta$ vs $r_{0}$ plane for systems with fixed electron number $N$ [see Figs. 2(a)-2(d)]. The insets in the figures give the single-particle picture representation for each phase. In the calculations, we took the confinement energy $\hbar \omega_{0}=5 \mathrm{meV}$. Several of the transitions between the phases can be understood from a single-particle (SP) picture when the exchange-correlation effects are added. The confinement in the $z$ direction splits the single QR levels (Fig. 1) into a set of bonding and antibonding levels by $\Delta$.

From such a SP picture, one does not expect configurations different from the CQDs as long as the level $\epsilon_{1,0}$ is not occupied. Indeed, for CQRs with $N \leqslant 8$ we found qualitatively similar ground states as for CQDs. For $N=8$, four different phases are found as shown in Fig. 2(a). For fixed $r_{0}$, there is a single ring atomic-type phase $(2,0,0)$ at small $\Delta$ where the inter-ring distance is large and the rings are decoupled. For large $\Delta$ the two rings are strongly coupled and act as a single one resulting in another atomic-type phase $(1,0,4)$. There are two molecular-type phases: $(0,0,2)$ and $(1,2,3)$ for intermediate inter-ring distance similar to those found earlier for CQDs. It is clear that, with decreasing $\Delta$, more electrons from the antibonding state transfer to the bonding state leading to phase transitions. Because the SP levels $\epsilon_{0, m}$ (for $m=0, \pm 1$, and \pm 2 ) decrease with increasing ring radius $r_{0}$ and converge at large ring radius, the atomictype phase $(1,0,4)$ of strongly coupled QRs is enhanced at large $r_{0}$. To this end, we can also understand that the tunneling effect is enhanced at large $r_{0}$. This becomes obvious when we investigate the phase transitions with increasing $r_{0}$ for a fixed $\Delta$. At $\Delta=2 \mathrm{meV}(d=32.8 \AA)$, e.g., the transitions $(0,0,2) \rightarrow(1,2,3) \rightarrow(1,0,4)$ correspond to the isospin transitions $I_{z}=2 \rightarrow 3 \rightarrow 4$ where the maximum value is reached for large $r_{0}$. Notice that the magnetic state, i.e., the $M_{z}$ value, can be manipulated by varying $\Delta$ and/or $r_{0}$.

Novel phases appear when $N \geqslant 9$. Let us first study the case of nine electrons [see Fig. 2(b)]. The phase $(3 / 2,0,9 / 2)$, for $r_{0}<0.89 \mathrm{a}_{0}$, is consistent with the result obtained for CQDs. ${ }^{15}$ A ring atomic-type phase $(1 / 2,2,9 / 2)$ 

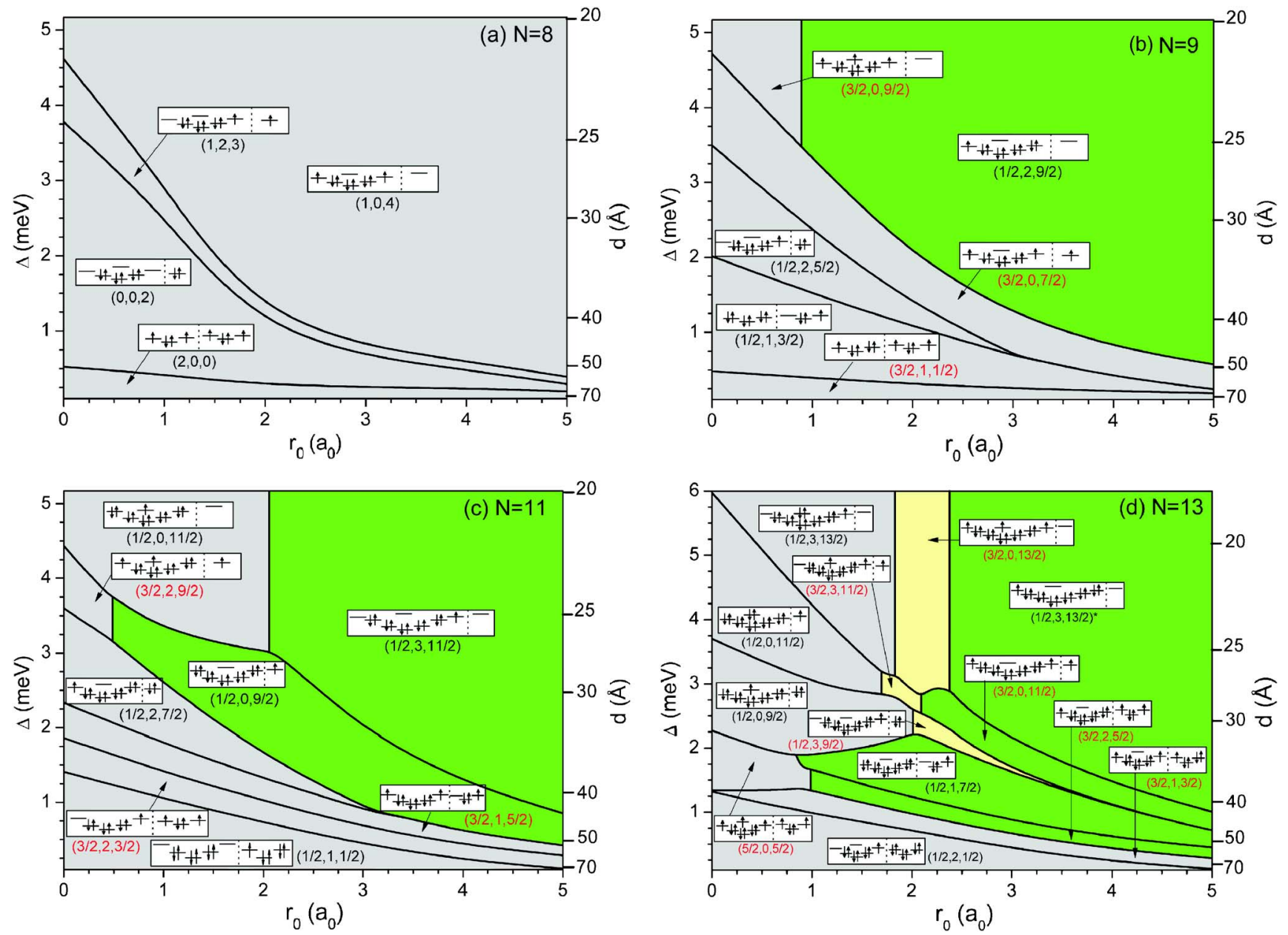

FIG. 2. (Color online) The ground-state phase diagrams of the AMs composed by CQRs in the plane of the inter-ring tunneling energy (inter-ring distance) vs the ring radius for (a) $N=8$, (b) $N=9$, (c) $N=11$, and (d) $N=13$ electrons. $\hbar \omega_{0}=5$ meV. The gray (green) region indicates the phases which can be found at $r_{0} \rightarrow 0\left(r_{0} \rightarrow \infty\right)$. The yellow region shows the phases which exist only for intermediate ring radii. The insets indicate the single-particle picture representation for each phase where the bonding (antibonding) states are represented on the left (right) of the vertical dotted line.

appears for $r_{0}>0.89 \mathrm{a}_{0}$ in the strong tunneling regime. The emergence of the new phase $(1 / 2,2,9 / 2)$ originates from the lifting of the degeneracy of the SP levels $\epsilon_{1,0}$ and $\epsilon_{0, \pm 2}$ in the bonding states. When the ring radius increases, $\epsilon_{1,0}-\epsilon_{0, \pm 2}$ increases and when this energy difference overcomes the exchange energy due to Hund's rule, the state $\epsilon_{0, \pm 2}$ becomes energetically more favorable than $\epsilon_{1,0}$. Consequently, a ground-state transition undergoes from $(3 / 2,0,9 / 2)$ to $(1 / 2,2,9 / 2)$.

Another result for the CQRs of nine electrons is the disappearance of the ground state $(1 / 2,2,5 / 2)$ for $r_{0}>3.04 \mathrm{a}_{0}$ which can be understood as follows: (i) the energy difference $\epsilon_{0, \pm 1}-\epsilon_{0,0}$ in the antibonding states is small at large $r_{0}$ (it is much smaller than $\epsilon_{0, \pm 2}-\epsilon_{0, \pm 1}$ ); and (ii) the existence of the $(3 / 2,0,7 / 2)$ phase is largely due to the exchange gain of the two aligned spin states in $\epsilon_{0,-2}$ and $\epsilon_{0,+2}$ in the bonding states. For $r_{0}>3.04 \mathrm{a}_{0}$, this exchange gain becomes larger than $\epsilon_{0, \pm 1}-\epsilon_{0,0}$. With decreasing $\Delta$, as soon as one of the electrons in the $\epsilon_{0, \pm 2}$ levels in the bonding states jumps to the antibonding state, the other electron follows leading to the transition from $(3 / 2,0,7 / 2)$ directly to the $(1 / 2,1,3 / 2)$ phase. This is a clear manifestation of the more pronounced effect of the electron-electron interaction in CQRs.

For 11 (and 12) electrons the number of possible ground states jumps to 8 [see Fig. 2(c)]. Among them, the ring atomic-type phase $(1 / 2,3,11 / 2)$ and the ring molecular-type phase $(1 / 2,0,9 / 2)$ appear only at finite ring radius. The transition from $(1 / 2,0,11 / 2)$ to $(1 / 2,3,11 / 2)$ at $r_{0}$ $=2.05 \mathrm{a}_{0}$ is associated with the transfer of a bonding state electron from $\epsilon_{1,0}$ to $\epsilon_{0, \pm 3}$. This is almost completely determined by the SP energy crossover of the two levels at $r_{0}$ $=2.07 \mathrm{a}_{0}$. We also notice that the molecular-type phase $(1 / 2,2,7 / 2)$ is completely suppressed at large ring radius $\left(r_{0}=3.17 \mathrm{a}_{0}\right)$. A similar phenomenon was already found for the $N=9$ case and is a consequence of the fact that the energy difference $\epsilon_{0, \pm 1}-\epsilon_{0,0}$ in the antibonding state at large $r_{0}$ becomes smaller than the exchange gain in the $(3 / 2,1,5 / 2)$ phase. We also observe a stabilization of the ring moleculartype phase $(1 / 2,0,9 / 2)$ at intermediate ring radius where the inter-ring tunneling energy $\Delta$ leads to a crossing of the lowest level $\epsilon_{0,0}$ in the antibonding state with the level $\epsilon_{0, \pm 3}$ in the bonding state. The lowest antibonding level $\epsilon_{0,0}$ is almost 
flat with increasing $r_{0}$ while the level $\epsilon_{0, \pm 3}$ decreases rapidly. As a consequence, the phase $(1 / 2,0,9 / 2)$ with one electron in the antibonding level $\epsilon_{0,0}$ is enhanced.

The number of possible phases increases to 13 for a $\mathrm{CQR}$ with 13 electrons [see Fig. 2(d)]. From left to right on the top of the figure, we see three phases: $(1 / 2,3,13 / 2)$, $(3 / 2,0,13 / 2)$, and $(1 / 2,3,13 / 2)^{*}$ where the star denotes that, in this new ring-type phase, the levels $\epsilon_{0, \pm 3}$ of higher angular momentum are occupied instead of the level $\epsilon_{1,0}$ in the quantum-dot-type phase $(1 / 2,3,13 / 2)$. The phase $(1 / 2,3,13 / 2)^{*}$ is due to the SP level $\epsilon_{0, \pm 3}$ which becomes lower in energy than the level $\epsilon_{1,0}$ in a quantum ring. The intermediate phase $(3 / 2,0,13 / 2)$ is due to many-body exchange-correlation effects. With decreasing $\Delta$, one of the electrons in the bonding state in the above configurations jumps to the lowest antibonding level leading to three molecular-type phases: $(1 / 2,0,11 / 2),(3 / 2,3,11 / 2)$, and $(3 / 2,0,11 / 2)$.

With further reducing the inter-ring tunneling energy, transitions occur from $(5 / 2,0,5 / 2)$ to $(3 / 2,2,5 / 2)$ at $r_{0}$ $=0.99 \mathrm{a}_{0}$ and to $(1 / 2,1,7 / 2)$ with increasing ring radius. These ring molecular-type phases are a consequence of the lifting of the degeneracy of the levels $\epsilon_{0, \pm 2}$ and $\epsilon_{1,0}$ at finite ring radius and results also in a more stable $(3 / 2,1,3 / 2)$ phase. The molecular-type phase $(1 / 2,3,9 / 2)$ turns to be unstable for $r_{0}=3.89 \mathrm{a}_{0}$ due to a strong exchange-correlation gain in its neighbor phase $(3 / 2,0,11 / 2)$. For CQRs of 13 electrons, we found that the following transitions are invoked by state changes of two electrons: $(1 / 2,0,9 / 2)$ to $(5 / 2,0,5 / 2), \quad(5 / 2,0,5 / 2) \quad$ to $(1 / 2,1,7 / 2), \quad$ and $(1 / 2,1,7 / 2)$ to $(3 / 2,0,11 / 2)$ where the total spin changes with $\left|\Delta S_{z}\right| \geqslant 1$.

Experimentally, it is more convenient to tune the number of electrons ${ }^{19}$ occupying the CQR. Therefore, we calculate the addition energy as a function of the electron number in strong, intermediate and weak coupling regimes as shown in Fig. 3. For $r_{0}=4.0 \mathrm{a}_{0}$ and $\Delta=1.6 \mathrm{meV}(d=35.8 \AA)$ in Fig. $3(\mathrm{a})$, the two rings are strongly coupled acting as a single one. For a single ring, the closed shells are formed ${ }^{10}$ at $N$ $=2,6,10, \ldots$, resulting in peaks in the addition energy. From the inset we notice that $I_{z}$ increases monotonously with increasing $N$ because all the electrons are in the bonding states. When we reduce the ring radius to $r_{0}=2.0 \mathrm{a}_{0}$ while keeping the same tunneling energy $\Delta=1.6 \mathrm{meV}$ as shown in Fig. 3(b), we find the CQRs entering the molecular-type phase for $N \geqslant 7$. The strong addition energy peak at $N=6$ indicates that the system transfers from a closed-shell ring atomic-type phase $(0,0,3)$ into a molecular-type phase $(1 / 2,0,5 / 2)$ for $N=7$ where there is one electron in the lowest antibonding level $\epsilon_{0,0}$. From $N=7$ to $8, I_{z}$ changes from $5 / 2$ to 4 as shown in the inset of Fig. 3(b) with a total isospin change $\left|\Delta I_{z}\right|$ $=3 / 2>1 / 2$ resulting in a strong reduced current peak ${ }^{15,19}$ because of isospin blockade. In this intermediate coupling regime, the ground-state phase depends strongly on both the ring radius and the inter-ring distance as shown in Fig. 2. This is clearly reflected in the addition energy. The peak in the addition energy at $N=12$ in Fig. 3(b) corresponds to the molecular-type phase $(0,0,4)$ where there are ten paired electrons in the bonding levels and two paired electrons in the antibonding level $\epsilon_{0,0}$.

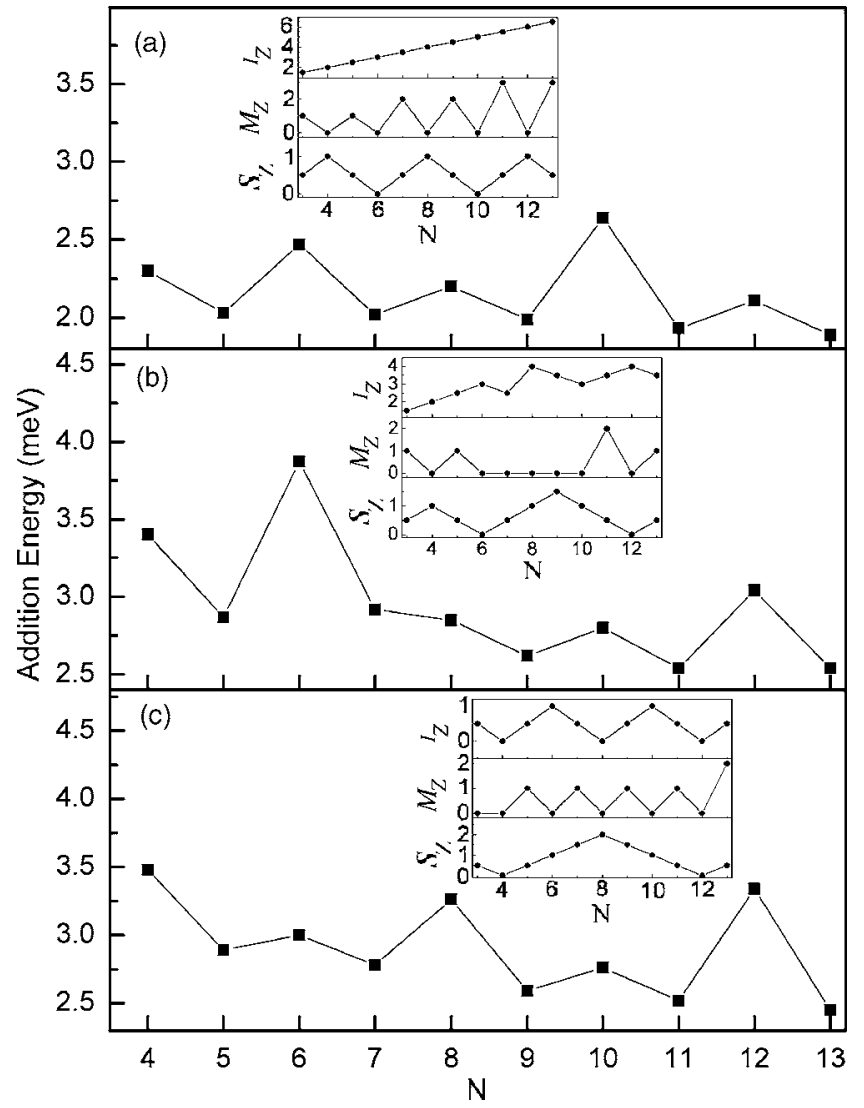

FIG. 3. The addition energy as a function of the number of electrons in the CQRs with (a) $r_{0}=4.0 \mathrm{a}_{0}$ and $\Delta=1.6 \mathrm{meV}$; (b) $r_{0}$ $=2.0 \mathrm{a}_{0}$ and $\Delta=1.6 \mathrm{meV}$; and (c) $r_{0}=2.0 \mathrm{a}_{0}$ and $\Delta=0.15 \mathrm{meV}$. The insets show the corresponding total spin $S_{z}$, angular momentum $M_{z}$, and isospin $I_{z}$.

When the ring radius is kept as a constant, the CQRs become weakly coupled for large inter-ring distance. Such a situation is presented in Fig. 3(c) for $r_{0}=2.0 a_{0}$ and $d$ $=67.4 \AA(\Delta=0.15 \mathrm{meV})$ where the CQRs are in the weakcoupling regime. Because the system evolutes to two separated rings at $d \rightarrow \infty(\Delta \rightarrow 0)$, one should find closed shells at $N=4,12,20, \ldots$, for single rings. We see these features in the inset in Fig. 3(c). However, we also find a pronounced addition energy peak at $N=8$ indicating coupling of two halffilled rings with total spin $S_{z}=2$. The peak at $N=12$ corresponds to two closed-shell rings of six electrons in each ring.

\section{CONCLUSIONS}

In summary, new quantum ring molecular-type phases, as well as ring atomic-type phases, are predicted for the CQRs which can be probed experimentally through current measurements. Our results show transitions of these artificial molecular phases from the CQDs $\left(r_{0}=0\right)$ to the CQRs and also from two strongly coupled quantum ring AMs to their dissociation in the weak tunneling regime. In comparison to the system of CQDs, quantum tunneling effects are enhanced in the present system where the AMs require less tunneling energy to enter the molecular-type phases. 


\section{ACKNOWLEDGMENTS}

This work was supported by FAPESP and CNPq (Brazil) and by the Flemish Science Foundation (FWO-Vl) and the Belgium Science Policy. Part of this work was supported by the EU network of excellence: SANDiE.
*Electronic address: hai@ifsc.usp.br

†Electronic address: francois.peeters@ua.ac.be

${ }^{1}$ S. M. Reimann and M. Manninen, Rev. Mod. Phys. 74, 1283 (2002).

${ }^{2}$ Aharonov-Bohm and other Cyclic Phenomena, J. Hamilton (Springer-Verlag, Berlin, 1997).

${ }^{3}$ R. Held, S. Lscher, T. Heinzel, K. Ensslin, and W. Wegscheider, Appl. Phys. Lett. 75, 1134 (1999); A. Fuhrer et al., Nature (London) 413, 822 (2001).

${ }^{4}$ J. M. García, G. Medeiros-Ribeiro, K. Schmidt, T. Ngo, J. L. Feng, A. Lorke, J. Kotthaus, and P. M. Petroff, Appl. Phys. Lett. 71, 2014 (1997); A. Lorke, R. J. Luyken, A. O. Govorov, J. P. Kotthaus, J. M. Garcia, and P. M. Petroff, Phys. Rev. Lett. 84, 2223 (2000).

${ }^{5}$ Z. Gong, Z. C. Niu, S. S. Huang, Z. D. Fang, B. Q. Sun, and J. B. Xia, Appl. Phys. Lett. 87, 093116 (2005).

${ }^{6}$ T. Mano, T. Kuroda, S. Sanuginetti, T. Ochiai, T. Tateno, J. Kim, T. Noda, M. Kawabe, K. Sakoda, G. Kido, and N. Koguchi, Nano Lett. 5, 425 (2005).

${ }^{7}$ D. Granados, J. M. Garcia, T. Ben, and S. I. Molina, Appl. Phys. Lett. 86, 071918 (2005).

${ }^{8}$ J. Simonin, C. R. Proetto, Z. Barticevic, and G. Fuster, Phys. Rev. B 70, 205305 (2004).

${ }^{9}$ M. Koskinen, M. Manninen, B. Mottelson, and S. M. Reimann,
Phys. Rev. B 63, 205323 (2001).

${ }^{10}$ J. C. Lin and G. Y. Guo, Phys. Rev. B 65, 035304 (2001).

${ }^{11}$ S. Viefers, P. S. Deo, S. M. Reimann, M. Manninen, and M. Koskinen, Phys. Rev. B 62, 10668 (2000).

${ }^{12}$ Y. Li and H. S. Lu, J. Phys. Soc. Jpn. 43, 2014 (2004).

${ }^{13}$ J. I. Climente and J. Planelles, Phys. Rev. B 72, 155322 (2005).

${ }^{14}$ B. Szafran and F. M. Peeters, Phys. Rev. B 72, 155316 (2005).

${ }^{15}$ B. Partoens and F. M. Peeters, Phys. Rev. Lett. 84, 4433 (2000).

${ }^{16}$ B. Tanatar and D. M. Ceperley, Phys. Rev. B 39, 5005 (1989).

${ }^{17}$ M. Pi, A. Emperador, M. Barranco, F. Garcias, K. Muraki, S. Tarucha, and D. G. Austing, Phys. Rev. Lett. 87, 066801 (2001).

${ }^{18}$ M. Koskinen, M. Manninen, and S. M. Reimann, Phys. Rev. Lett. 79, 1389 (1997); M. Ferconi and G. Vignale, Phys. Rev. B 50, 14722 (1994); K. Hirose and N. S. Wingreen, Phys. Rev. B 59, 4604 (1999); O. Steffens and M. Suhrke, Phys. Rev. Lett. 82, 3891 (1999); S. Nagaraja, J. P. Leburton, and R. M. Martin, Phys. Rev. B 60, 8759 (1999); I.-H. Lee, V. Rao, R. M. Martin, and J. P. Leburton, Phys. Rev. B 57, 9035 (1997); M. Stopa, Phys. Rev. B 54, 13767 (1996).

${ }^{19}$ Y. Tokura, D. G. Austing, and S. Tarucha, J. Phys.: Condens. Matter 11, 6023 (1999); D. G. Austing, S. Tarucha, H. Tamura, K. Muraki, F. Ancilotto, M. Barranco, A. Emperador, R. Mayol, and M. Pi, Phys. Rev. B 70, 045324 (2004). 